\title{
The silent killer: myocardial injury after non-cardiac surgery (MINS)
}

\author{
Simpson GC11, Marais LC², Rodseth RN³, Outcomes Research Consortium
}

${ }_{1}$ MBChB, DA(SA); Anaesthetic registrar, Nelson R Mandela School of Medicine, University of KwaZulu-Natal, Pietermaritzburg, South Africa

2 MBChB, FC Orth SA, MMed(Ortho), PhD; Department of Orthopaedics, School of Clinical Medicine, University of KwaZulu-Natal, Pietermaritzburg, South Africa

${ }^{3}$ MBChB, FCA, Cert Crit Care, MMed, MSc, PhD; Perioperative Research Group, Department of Anaesthetics, Grey's Hospital, Nelson R Mandela School of Medicine, University of KwaZulu-Natal, Pietermaritzburg, South Africa

${ }^{4}$ Cleveland, Ohio

Corresponding author: Dr Gary Simpson; Grey’s Tertiary Hospital, Department of Anaesthetics, Critical Care and Pain; email: drgcsimpson@gmail.com; cell: 0837958712

\begin{abstract}
Introduction: Recent work into the causes of death after non-cardiac surgery has identified a new clinical concept, namely myocardial injury after non-cardiac surgery (MINS). The pathophysiology is related to a supply-and-demand mismatch in the peri-operative period and differs from the traditional model of myocardial ischaemia and infarction.

Methods: Literature review of current body of knowledge and recent large multicentre clinical trials.

Results: MINS is associated with increased morbidity and mortality at 30 days' post-surgery. A large international multicentre trial found that a troponin T level of greater than $0.3 \mathrm{ng} / \mathrm{ml}$ was associated with a mortality rate of $16.9 \%$. Moreover, $84.2 \%$ of MINS would probably go undetected if systematic troponin monitoring after surgery was not performed.
\end{abstract}

Conclusion: This review examines the current body of knowledge and provides practical guidelines on how to identify and manage patients with MINS.

Level of evidence: Level 5

Key words: MINS, myocardial injury after non-cardiac surgery, troponin elevation, peri-operative myocardial injury, myocardial ischaemia

Citation: Simpson GC, Marais LC, Rodseth RN, Outcomes Research Consortium. The silent killer: myocardial injury after non-cardiac surgery (MINS). SA Orthop J 2018;17(2):12-15. http://dx.doi.org/10.17159/2309-8309/2018/v17n2a1

Editor: Prof Anton Schepers, University of the Witwatersrand
Received: May 2017
Accepted: July 2017
Published: May 2018

Copyright: ( 2018 Simpson GC, et al. This is an open-access article distributed under the terms of the Creative Commons Attribution Licence, which permits unrestricted use, distribution and reproduction in any medium, provided the original author and source are credited.

Funding: No funding was received for this review article.

Conflict of interest: The authors declare they have no direct or indirect conflict of interests related to this review article. 


\section{Introduction}

Pulmonary embolism (PE) is frequently cited as the prime suspect in the case of an unexpected post-operative mortality. However, this belief may be unfounded as a new culprit has recently been identified as a major cause of post-operative morbidity and mortality.

From 1966 to 1986 PE and deep vein thrombosis (DVT) had a reported baseline mortality of $0.1-0.8 \%$ and, when associated with hip fractures, a mortality of $7 \%$. To put it into perspective, if Moses Mabhida Stadium in Durban was filled to its capacity of 85000 spectators, 6000 would have perished due to DVT/PE. Fast forward to 2014 and the Vascular Events in Noncardiac Surgery Patients Cohort Evaluation (VISION study), which enrolled over 15000 participants undergoing non-cardiac surgery, recorded a DVT/PE rate of $0.6 \%$ and mortality of $0.07 \%$. In comparison to the 6000 spectators in Moses Mabhida Stadium that would have succumbed, this number has reduced to 60.1-3 This dramatic reduction can be attributed to increased vigilance and carefu attention to mechanical and chemical DVT prophylaxis. ${ }^{4}$ However, although DVT/PE remains an important differential diagnosis of postoperative morbidity and mortality, it seems that we need to continue looking.

Recent work into the causes of death after non-cardiac surgery has identified a new clinical concept - myocardial injury after noncardiac surgery (MINS) - that is strongly associated with adverse post-operative events. Data from the VISION study showed that an elevated troponin level after non-cardiac surgery, irrespective of the presence of ischaemic features or clinical symptoms, was an independent predictor of 30-day mortality. ${ }^{2}$ MINS clearly is an important warning sign that warrants our attention.

This review examines the current body of knowledge and provides practical guidelines on how to identify and manage patients with MINS. This review also aims to encourage a change in basic assumptions, and it emphasises the need to actively look for other treatable insults to our patients.

\section{MINS}

MINS is a unique sub-clinical diagnosis that does not meet the clinical criteria traditionally used to diagnose myocardial injury. MINS is an under-recognised entity among adults undergoing noncardiac surgery and associated with substantial mortality. ${ }^{2}$ The pathophysiology is related to a supply-and-demand mismatch in the peri-operative period and differs from the traditional model of myocardial ischaemia secondary to vessel occlusion by thrombus or ruptured plaque.

In contrast with the traditional concept of a myocardial infarction (MI) which presents with the triad of: clinical symptoms; ECG changes; and a troponin elevation higher than the 95th centile, MINS requires us to think differently. With MINS, even a minor troponin elevation, with or without chest pain or ECG changes, significantly increases the risk of post-operative death. Mortality following surgery without evidence of MINS is approximately 1\%. This increases to $9.5 \%$ in the presence of MINS. The risk of congestive heart failure, stroke and non-fatal $\mathrm{Ml}$ increased from $2.4 \%$ to $18.5 \%$ in the presence of MINS. ${ }^{2}$ Experience at our local tertiary level training hospital reveals a two-year mortality rate close to $28 \%$ in the presence of MINS, that is, one in three patients diagnosed with MINS will be dead within two years of the event.

An excellent article published in the journal Anesthesiology, that is based on analysis of the VISION data, illustrates many of the unique features of MINS. ${ }^{2}$ The definition of MINS is suggested as 'prognostically relevant myocardial injury due to ischaemia that occurs within 30 days post-operatively'. This research group examined data from 15065 patients from North America, South America, Europe, Africa and Asia with a follow up of $99.7 \%$ at 30 days. The focus of many studies on cardiac complications following non-cardiac surgery is on peri-operative myocardial infarction as diagnosed by the universal definition. VISION found that if the universal definition was used alone, $58.2 \%$ of patients with prognostically significant myocardial injury would be missed. Moreover, $84.2 \%$ of MINS would probably go undetected if systematic troponin monitoring after surgery was not performed. Table I summarises key numbers of patients affected by MINS.

\section{Table I: Key numbers of myocardial injury after non-cardiac surgery (MINS)}

$8.0 \%$ of patients over 45 years of age undergoing non-cardiac surgery suffer MINS.

Worldwide, 100 million adults over 45 years of age undergo non-cardiac surgery; this suggests that 8 million adults suffer from MINS.

6.75 million adults suffering from MINS would go undetected if not systematically screened for.

1 in 10 patients suffering from MINS will die within 30 days.

Evaluating patients for MINS compared with the traditional diagnosis of myocardial infarction will allow the treating clinician to avoid missing most patients with prognostically significant perioperative myocardial injury.

\section{Pre-operative screening and prevention}

In a South African setting, where pathology consistently presents late, we now have the means to detect myocardial injury at the subclinical stage and provide treatment that will improve patient outcomes.

The Canadian Cardiovascular Society Guidelines on Perioperative Cardiac Risk Assessment and Management for Patients Who Undergo Noncardiac Surgery (CCS Guidelines) is a comprehensive updated guideline released in 2016 that shifts the focus to first identify 'at risk' patients and secondly to initiate early intensive therapies for myocardial injury. ${ }^{5}$

The CCS Guidelines recommend measuring brain natriuretic peptide (BNP) or N-terminal fragment of proBNP (NT-proBNP) before surgery to enhance peri-operative cardiac risk estimation in patients who are 65 years of age or older and in patients who are 45-64 years of age with significant cardiovascular disease or in patients with an increased Revised Cardiac Risk Index Score (RCRI Score). The CCS Guidelines also importantly advise against extensive pre-operative cardiac testing to enhance cardiac risk estimation.

Pre-operative testing has not been shown to improve outcomes beyond simple scoring with the RCRI Score. Many exercise stress tests are limited in patients with musculoskeletal pathology as they are simply unable to mechanically complete the stress test, and therefore these tests offer little insight into the cardiovascular reserves of these patients. Extensive pre-operative cardiovascular investigations often delay surgery and add little to the management of patients; these tests are also often unavailable at many institutions. Only specific cardiovascular diseases require pre-operative testing. An example may include pre-operative $\mathrm{ECHO}$ for valvular heart disease.

QxMd or QxCalculate is a smartphone application that is freely available for download which has the RCRI Score preloaded. You are required to select answers for six questions, and then the app calculates the risk. The questions are: 1) High risk surgery? 2) Coronary artery disease? 3) Congestive heart failure? 4) Cerebrovascular disease? 5) DM on insulin? 6) Serum creatinine $>177 \mathrm{mcg} / \mathrm{l}$ ? This score is used for discussion with patients regarding potential risk, peri-operative planning, and post-operative screening.

Our patients may already be receiving treatment for their comorbidities and, in addition to cardiovascular screening, the patient's cardiovascular medication may need to be adjusted in the 
peri-operative period. It is now established that starting beta blockers pre-operatively reduces adverse cardiac events but increases cerebrovascular events and mortality. ${ }^{6}$ The current recommendations are to stop aspirin three days before surgery (to prevent bleeds) unless the patient has had a recent cardiac stent or is having carotid surgery; stop angiotensin-converting enzyme inhibitors (ACEI) 24 hours prior to surgery; to start statins daily (three to seven days before surgery); and to continue beta blocker therapy (if already receiving beta blocker) including on the day of surgery.

\section{Post-operative surveillance and treatment}

MINS is associated with increased morbidity and mortality at 30 days post-surgery. In 2012, original research from the VISION group evaluated 15133 patients and found that elevated troponin levels as well as measuring peak troponin levels added prognostic value and enabled the clinician to discriminate those likely to die in 30 days. $^{7}$ Identifying and treating patients with a troponin leak or MINS can ensure that we send more patients home alive. The CCS guidelines recommend measuring daily troponins for 48-72 hours after surgery in high risk patients (these are patients over 45 years of age with comorbidities or older than 64 years of age). They recommend initiating aspirin and statin therapy in patients who suffer myocardial injury after surgery.

The advancement and availability of biomarkers has allowed us to incorporate current research and best practices into daily clinical practice. Biomarkers allow us to diagnose sub-clinical myocardial injury and act on these diagnoses. While troponin $\mathrm{T}$ levels higher than $0.03 \mathrm{ng} / \mathrm{ml}$ are considered diagnostic of MINS, ${ }^{2}$ a troponin T level of greater than $0.3 \mathrm{ng} / \mathrm{ml}$ had a mortality rate of $16.9 \% .^{8}$ The likelihood ratio for peak troponin T measurement above $0.03 \mathrm{ng} / \mathrm{ml}$ was 5.39 and measurements above $0.3 \mathrm{ng} / \mathrm{ml}$ was 10.71. The likelihood ratio is the likelihood that a given test result would be expected in a patient with the target disorder compared to the likelihood that that same result would be expected in a patient without the target disorder, where a likelihood ratio of greater than 1 indicates the test result is associated with the disease and increasing values represent increasing probability of the disease. Therefore, what was previously considered a minor troponin elevation of troponin $\mathrm{T} \geq 0.03 \mathrm{ng} / \mathrm{ml}$ or high sensitivity troponin $\mathrm{T}$ levels above $0.06 \mathrm{ng} / \mathrm{ml}$ now allows the

\section{Protocolformajor surgical peri-operative management} Step 1 - Score (using QxMD.com)

Revised Cardiac Risk Index - using QXMD.com/calculate

Use protocol if: $\geq 64$ years, or $\geq 45$ years old significant cardiovascular disease, or $R C R I \geq 2$

\begin{tabular}{|c|c|}
\hline Step 2 - Pre-operative prep & Done? \\
\hline Patient & \\
\hline Pre-operative BNP & \\
\hline Baseline ECG & \\
\hline Baseline CXR & \\
\hline Pre-operative echo (if clinical signs of valvular disease, pulmonary hypertension or HOCM) & \\
\hline Lung function tests (if diagnosed COPD) & \\
\hline Drugs & \\
\hline Aspirin-stop3days beforesurgery(unlessrecentcardiacstentorhaving carotidsurgery) & \\
\hline ACE - stop 24 hours before surgery & \\
\hline $\begin{array}{l}\text { Statin-40 mg simvastatin daily or } 40 \mathrm{mg} \text { atorvastatin daily 3-7 days before surgery. Startifnot taking orincrease dose ifalready on } \\
\text { it. Takeonmorning of surgery }\end{array}$ & \\
\hline Beta-blocker-Continue if on beta-blocker (unless systolic $B P<95 \mathrm{mmHg}$ ). Take on morning of surgery. & \\
\hline Logistics & \\
\hline Submit ICU consult 2 working days before surgery & \\
\hline Submit anaesthetic consult 72 hours before surgery & \\
\hline Cross-match blood as per protocols & \\
\hline
\end{tabular}

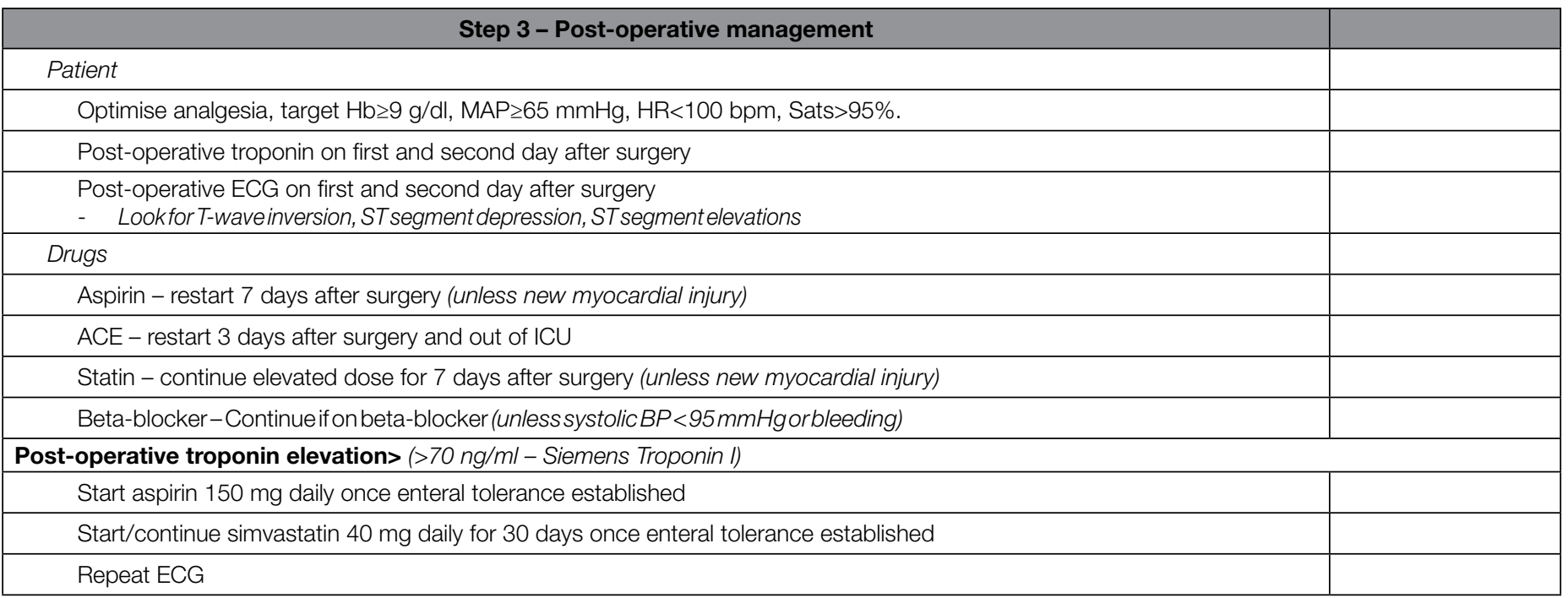

Figure 1: An example of a proposed peri-operative algorithm for high-risk patients, incorporating screening, detection and treatment guidelines for MINS (with permission from Grey's Department of Anaesthesia, Critical Care and Pain, KZN, RSA) 
diagnosis of MINS to be made. Starting aspirin daily together with starting or continuing a higher dose statin for 30 days and optimising any other comorbidities has been shown to improve outcomes. ${ }^{9}$

The long-term impact of early cardiovascular therapy intensification for post-operative troponin elevation has previously been evaluated. ${ }^{9}$ Foucrier et al. hypothesised that providing appropriate secondary preventative treatment to patients with elevated troponin levels would limit long-term mortality. The main finding was that patients with elevated troponin levels after non-cardiac surgery who did not receive intensified cardiovascular therapies were at a much higher risk for a major cardiac event; however, this study was looking at troponin elevation following vascular surgery specifically.

Taking this into account, the ideal post-operative management should include:

- optimising analgesia

- targeting haemoglobin concentrations $>9 \mathrm{~g} / \mathrm{dl}$

- targeting mean arterial pressure (MAP) $>65 \mathrm{mmHg}$, pulse rate less than $100 \mathrm{bpm}$ and saturation >95\%

- evaluating troponin I levels on the first and second day postoperatively

- post-operative ECGs on the first and second day after surgery looking for T-waves and ST segment changes

- restarting aspirin on day 8 post-surgery (unless MINS/new MI); restarting angiotensin-converting enzyme inhibitor (ACEI) after three days; continuing with elevated dose of statins for seven days (unless MINS/new MI); and continuing beta blocker therapy (unless systolic blood pressure is less than $95 \mathrm{mmHg}$ or bleeding).

Figure $I$ is a proposed pragmatic example currently being used in a tertiary hospital in KZN, RSA, to assess and optimise high risk patients prior to surgery.

\section{Further considerations}

MINS differs in pathophysiology when compared to myocardial ischaemia. The universal definition defines myocardial infarction as myocardial necrosis with three diagnostic criteria: clinical symptoms; elevated troponins above the 95th centile; and ECG changes in keeping with new ischaemia. The pathophysiology of the two entities are different and we therefore cannot readily apply treatment known to be effective in one setting to the other. However, by using evidence-based medicine recommendations for treatment of acute coronary syndromes we can hope to improve outcomes following the diagnosis of MINS. ${ }^{9}$

It should be noted that the analysis of the VISION data specifically excluded non-cardiac causes of elevated troponins. The treating physician must ensure non-cardiac causes of troponin leak are sought and excluded. Renal failure, sepsis and burns are common examples of non-cardiac aetiologies causing an elevation of troponin levels.

The associated costs of post-operative troponin surveillance and treatment in South Africa were evaluated by Biccard et al. ${ }^{8}$ They performed a pharmaco-economic analysis of routine troponin surveillance. The introduction of statin and aspirin therapy in patients who are troponin positive to reduce vascular mortality and perioperative myocardial infarction was found to be cost-effective.

Although no randomised controlled trials have established an effective treatment of MINS, it is suggested that the prognosis may be altered by applying the high-quality evidence for the use of aspirin and statins in treating myocardial injury in the non-surgical setting, as well as the body of evidence from therapy intensification after vascular surgery. ${ }^{9}$

Evidence presented in 2014 does show that troponin-directed therapy improves outcomes. The authors concluded, 'long-term adverse cardiac outcomes may likely be improved by following evidence-based recommendations for the medical management of acute coronary syndromes'. ${ }^{9}$

\section{Conclusion}

MINS is common and one in ten patients with MINS will die within 30 days of surgery. Failure to monitor for troponin elevation will result in more than $80 \%$ of MINS events remaining undetected. While focus should be maintained on being vigilant in monitoring and the prevention of pulmonary embolism, focus should also be on the prevention, early identification, and treatment of MINS. Increased awareness about MINS will likely improve outcomes and decrease peri-operative morbidity and mortality.

\section{Ethics statement}

The large multicentre clinical trials referenced in this literature review all received the required ethical permission from relevant local bioethics and research bodies. This literature review was not subject to the local BREC review.

\section{References}

1. Kakkar VV, Adams PC. Preventive and therapeutic approach to venous thromboembolic disease and pulmonary embolism - can death from pulmonary embolism be prevented? Journal of the American College of Cardiology. 1986;8(6):146B-58B.

2. Botto F, Alonso-Coello P, Chan MT, Villar JC, Xavier D, Srinathan $\mathrm{S}$, et al. Myocardial injury after noncardiac surgery: a large, international, prospective cohort study establishing diagnostic criteria, characteristics, predictors, and 30-day outcomes. Anesthesiology. 2014;120(3):564-78.

3. Shepard RM, White HA, Shirkey AL. Anticoagulant prophylaxis of thromboembolism in postsurgical patients. The American Journal of Surgery. 1966;112(5):698-702.

4. Guyatt GH, Akl EA, Crowther M, Gutterman DD, Schuunemann $\mathrm{HJ}$, American College of Chest Physicians Antithrombotic T, et al. Executive summary: Antithrombotic Therapy and Prevention of Thrombosis, 9th ed: American College of Chest Physicians Evidence-Based Clinical Practice Guidelines. Chest. 2012;141(2 Suppl):7S-47S

5. Duceppe E, Parlow J, MacDonald P, Lyons K, McMullen M, Srinathan S, et al. Canadian Cardiovascular Society Guidelines on Perioperative Cardiac Risk Assessment and Management for Patients Who Undergo Noncardiac Surgery. Can J Cardiol. 2017;33(1):17-32.

6. Devereaux PJ, Yang H, Yusuf S, Guyatt G, Leslie K, Villar JC, et al. Effects of extended-release metoprolol succinate in patients undergoing non-cardiac surgery (POISE trial): a randomised controlled trial. Lancet (London, England). 2008;371(9627):1839-47.

7. Devereaux PJ, Chan MT, Alonso-Coello P, Walsh M, Berwanger O, Villar JC, et al. Association between postoperative troponin levels and 30-day mortality among patients undergoing noncardiac surgery. JAMA. 2012;307(21):2295-304.

8. Biccard BM, Devereaux PJ, Rodseth RN. Cardiac biomarkers in the prediction of risk in the non-cardiac surgery setting. Anaesthesia. 2014;69(5):484-93

9. Foucrier A, Rodseth R, Aissaoui M, Ibanes C, Goarin JP, Landais $\mathrm{P}$, et al. The long-term impact of early cardiovascular therapy intensification for postoperative troponin elevation after major vascular surgery. Anesthesia and Analgesia. 2014;119(5):1053-63. 